\title{
Past Experiences and Attitude on Vaccination
}

\author{
Tandiyo Pradekso ${ }^{1}$, S. Rouli Manalu ${ }^{2}$, Djoko Setyabudi ${ }^{3}$ \\ \{tandiyop@yahoo.com $\left.{ }^{1}\right\}$ \\ Universitas Diponegoro, Indonesia ${ }^{1,2,3}$
}

\begin{abstract}
The Covid-19 vaccine is expected to be available in the next few months. There is hope and optimism in welcoming the upcoming availability of the vaccine, but at the same time there is also concern that there will be resistance from some groups in society who do not believe in using vaccines to prevent virus transmission and the emergence of disease. Reluctance to accept this vaccine is known as the vaccine hesitancy. The study was based on the theory of planned behavior (TPB) which explains that intention is predicted by the positive attitudes toward a specific behavior. This theory is generally used to predict the adoption rate of various health-related behaviors, including attitudes towards vaccination. The study reveals that past experiences of vaccination tend to illustrate that there are positive attitudes to vaccination among mothers surveyed. However, the focus should be directed to the parts that display barriers, drawbacks, or deficiencies, even only consists of small shares of the data. These small fragments could establish into the seed of vaccine hesitancy, which might have impacts on the efficacy of the upcoming Covid-19 vaccination.
\end{abstract}

Keywords: Vaccine Hesitancy, Vaccination

\section{Introduction}

Vaccines and vaccinations are two popular terms in the recent circumstances of the Covid-19 pandemic that is currently all-encompassing the world, including in Indonesia. People are becoming more comfortable to hearing the terms that were previously understood mostly by mothers of raising their young children. In the midst of the current pandemic situation, the Covid-19 vaccine is expected to be available in the next few months. The availability of the Covid-19 vaccine should swiftly overcome the outbreak that has threatening the human life. There are hopes and optimism in welcoming the upcoming availability of the vaccine, but at the same time there is also concern that there will be resistance from some groups in society who do not believe in using vaccines to prevent virus transmission and the emergence of disease. Reluctance to accept this vaccine is known as the vaccine hesitancy. Although a lot of medical and scientific evidence has shown that the use of vaccines can prevent disease and save millions of lives (especially children) each year, the symptoms of vaccine rejection in some groups of society continue to occur. The World Health Organization (WHO) has even placed vaccine hesitancy as one (number 8 ) of the 10 of biggest threats to world health in 2019 [1]. In Indonesia itself, the 2018 data provided by the Welcome Global Monitor show that there are still around 11 percent of the population who do not believe in the effectiveness of vaccines [2].

A number of studies have shown that there are various reasons why vaccine hesitancy occurs. The reasons for hesitancy can be due to considerations of the type of vaccine, certain 
people or groups who refuse the vaccine, and the context of the vaccine. The diversity of reasons for vaccine hesitancy has been mapped by a WHO task force called the Strategic Advisory Group on Experts (SAGE) on Immunization [3]. Based on these maps they defined vaccine hesitancy as delaying or refusing vaccination even though vaccination services were available. It was further explained that vaccine hesitancy is complex and contextual, varying based on time, place, and type of vaccine. Vaccine hesitancy is influenced by various factors such as compliance, convenience and confidence. Furthermore, Larson [3] explains that although vaccine hesitancy occurs only in certain specific groups in society and does not extend to a large number of the population, it is very important to understand who are reluctant to receive vaccinations, why, and where they are. So, they can be identified by their geographical conditions, political contexts, and socio-cultural contexts that have something to do to vaccine hesitancy. Everything needs to be understood in order to find the best way to minimize hesitancy. Incomplete vaccination in a community will cause a potential breach that can thwart the achievement of herd immunity in that community. Mitigation of these problems will help develop effective strategies to reduce vaccine hesitancy and improve vaccine acceptance.

This article stems from a research that was based on the theory of planned behavior (TPB) which explains that intention is predicted by the positive attitudes toward a specific behavior, the approval of the other significant subjective norms and a perceived behavioral control. This theory is generally used to predict the adoption rate of various health-related behaviors, including attitudes towards vaccination. In its implementation, TPB is also integrated with a number of concepts including risk perception, past behavior, knowledge, and experience when used to analyze barriers to vaccination attitudes and behavior.

\section{Research Method}

The research was conducted through an online survey and invited the participation of 178 women who have children under five in Indonesia. Data analysis was conducted descriptively to explain various variables of past behavior and attitudes towards vaccination. Those who participated were aged between 19 and 52 years. Have an education level from junior high to graduate degree. Their daily activities also varied, including housewife $(47.8 \%)$, employees (24.2\%), self-employed (12.9\%), and professionals (10.1\%). Most participants have only one child under 5 years old (79.2\%), some have 2 children (15.2\%), and $61.2 \%$ do not have any child above the age of 5 . 


\section{Result and Discussion}

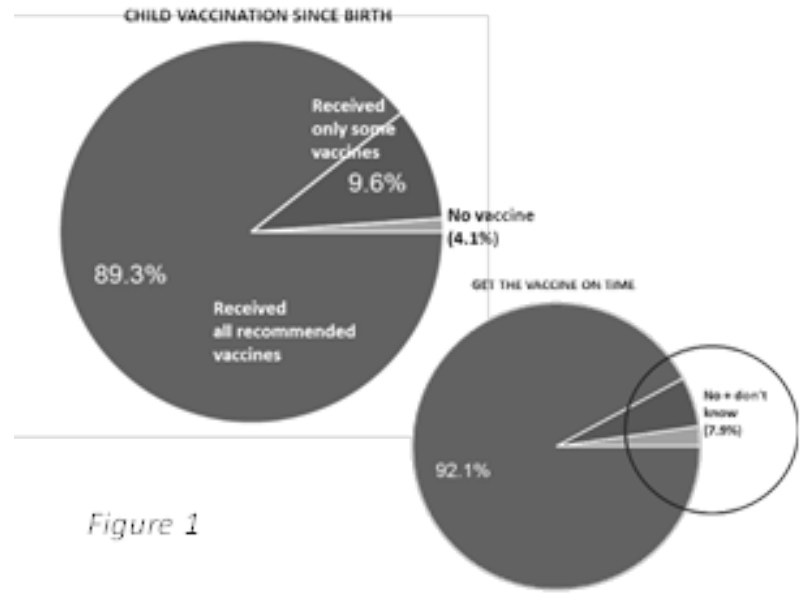

Responses from the survey participants revealed that most of their children had received all the recommended vaccines since birth. However, the slice of those who did not complete the vaccination and did not vaccinate their children was still above 10 percent. It is a significant figure considering the participants are urban mothers and familiar to internet-based communication routine a profile that usually associated to advancement of accessing a wide range of information. This share is like confirming the data from the Welcome Global Monitor which reveals that 11 percent of the population in Indonesia does not believe in the effectiveness of vaccination. The subsequent data also exposes that around 8 percent of participants do not even know when to do the vaccination to their children or do not vaccinate their children in time. Barriers and attitudes on vaccination.

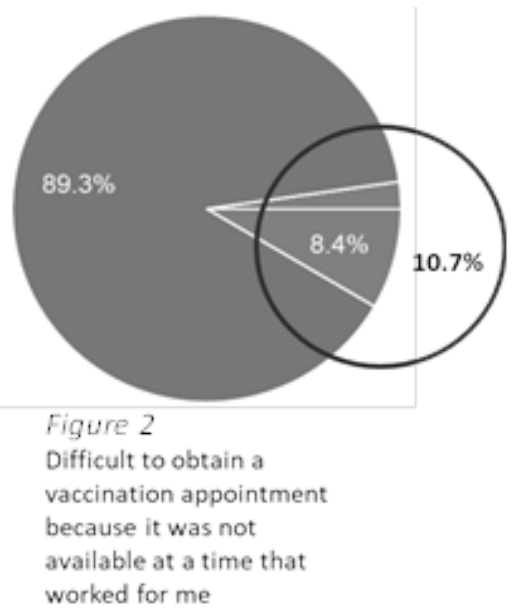

Altogether, from an optimistic point of view, we can have the confidence to argue that most mothers (approximately $90 \%$ ) do not face any barrier when vaccinating their children. However, as previously explained, vaccine hesitancy will never involve the majority portion, 
although its effects can affect the majority population. With this standpoint, an important part of detecting seeds of hesitancy is to look at the size of the small part who feel inhibited from vaccinating. Figure 2 shows that the problem of time that can be assigned by mothers to vaccinate their children is still an obstacle for some of them (especially mothers who work tight hours). More than 10 percent felt that they had difficulty managing their availability to the time scheduled by the clinic or vaccine provider. This is particularly occurred for the later phases of vaccinations when mothers have come out from their maternity break and breastfeeding, and back to their daily routines.

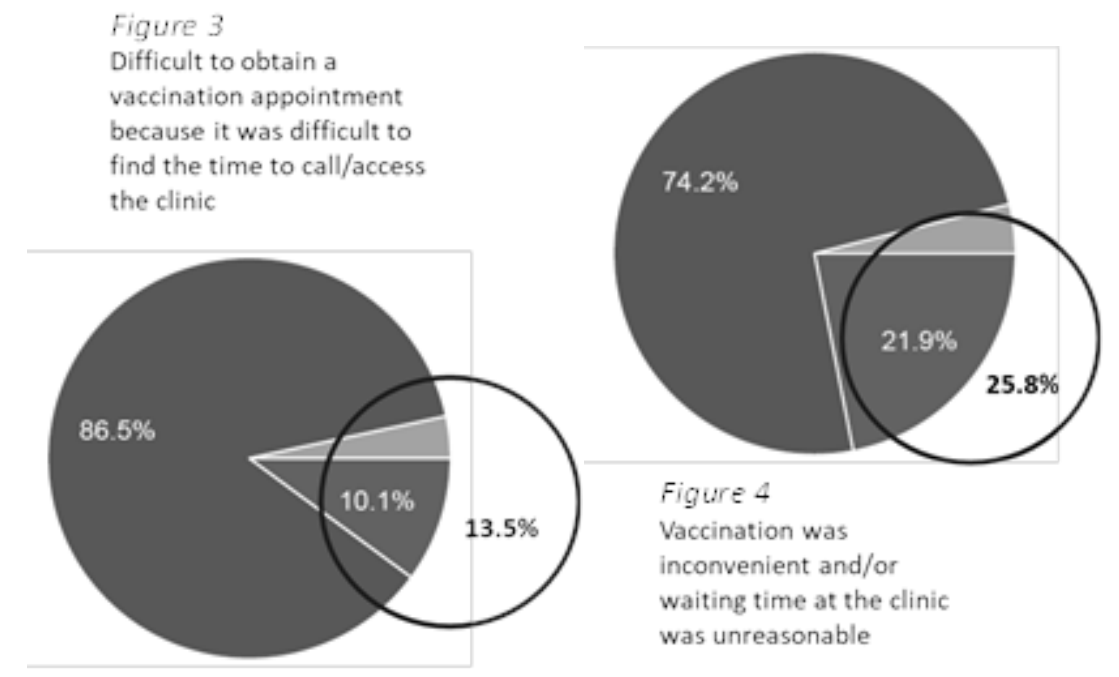

On the other hand, there are even more mothers (Figure 3) who find it difficult to access medical facilities and clinics that provide vaccination services. In this case, the barriers lie on the vaccine provider side that prevent or complicate mothers to access vaccines for their children. This response actually raises a big question. So far, vaccination services, especially for babies and children, have always been given the high priority by the authority. The frontline for infant and child vaccination services has reached almost all corners of the country. So, the fact of a fairly large proportion to the issue of access to vaccination will be a drawback for vaccination achievements. The complication felt by more mothers relates to the quality of vaccination services (Figure 4). The poor services are mostly due to the inconveniency or/and the waiting time at the vaccine providers that was distressing. Closely to 26 percent of respondents considered that situation was disturbing, unenthusiastic, and discouraging. In fact, vaccination services for children are provided in several types and levels. The government support vaccination services are delivered in community clinics (Puskesmas) and community health groups (Posyandu), and all the services are provided for free other than those are vaccination services provided by private hospitals, private doctors, and private midwives. People have to pay to obtain services from those private sources. 


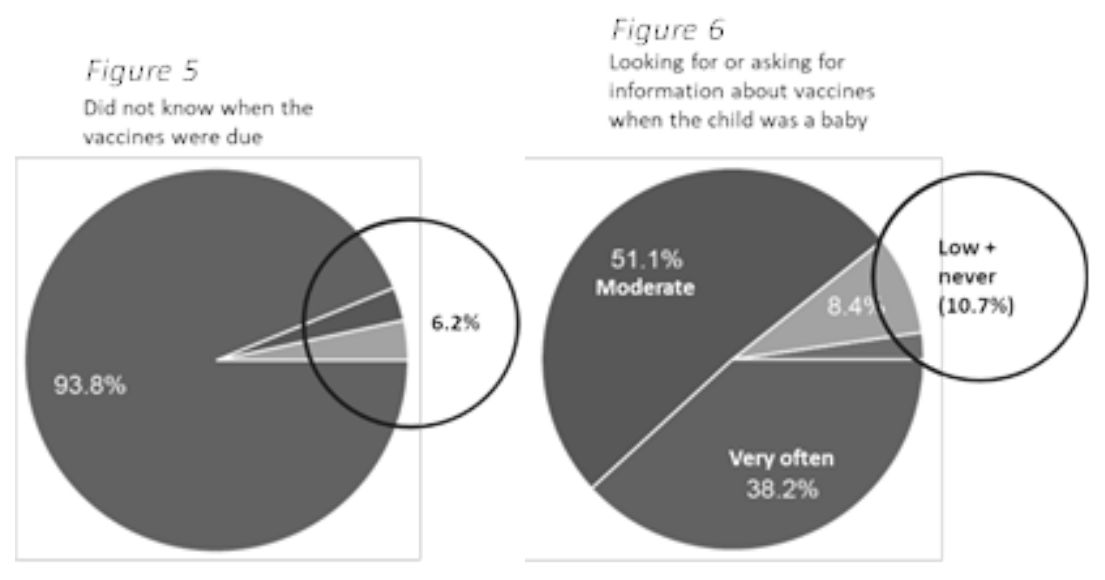

This study also found that there are still quite a number of mothers who are not sufficiently involved cognitively and practically in their children's vaccination activities. There are still around 6 percent of respondents who are not aware of the vaccination schedule for their children, and this lack of knowledge could contribute to the break of effective vaccination program. Concern about vaccination issues were varies among mothers surveyed. While approximately half of them moderately seeking further information about vaccines and vaccinations, a significant fragment inadequately put any effort to understand the issue. There were more than 10 percent of respondents who rarely and never try to find out about vaccines and vaccinations (Figure 6).

Overall, the study reveals that past experiences of vaccination tend to illustrate that there are positive attitudes to vaccination among mothers surveyed. However, the focus should be directed to the parts that display barriers, drawbacks, or deficiencies, even only consists of small shares of the data. These small fragments could establish into the seed of vaccine hesitancy, which might have impacts on the efficacy of the upcoming Covid-19 vaccination.

\section{Conclusion}

The study reveals that even though the prevalence rate of vaccinations among respondents is significantly high, concerns should be directed to those who (for a variety of reasons) are not reached by vaccination services. Findings further expose that access to and quality of vaccination services are significant precursors that could contribute to the mess of vaccination program. Knowledge and intention to vaccination are also essential and should be helpful in vaccination decision. Finally, there still some barriers and obstacles of preventing vaccination that need to be scrutinized, to develop a better way to promote vaccination.

\section{References}

[1] J. Wood, "Anti-vaccine sentiment one of 10 biggest health threats, says WHO," 2019.

[2] "Wellcome Global Monitor: How does the world feel about science and health?," 2019.

[3] J. Larson-Hall, A guide to doing statistics in second language research using SPSS and R. Routledge, 2015. 